\title{
Visualization of fire brigade interventions based on statistical data
}

\author{
Łukasz Wielebski ${ }^{\text {a, * }}$ \\ ${ }^{a}$ Affiliation: Department of Cartography and Geomatics, Adam Mickiewicz University, Poznan, Poland \\ lukwiel@amu.edu.pl,** \\ Corresponding author
}

Keywords: fire brigade intervention, cartographic visualizations, mapping techniques, statistical data, graphically supported analysis, effectiveness and usefulness

Graphically supported analysis of data concerning the intervention of selected Polish fire brigade units in the spatial and temporal depiction, as well as in the context of the recognition of the type and scale of threats that occur on the areas protected by those units, was the fundamental issue touched upon in the research. Statistical data from 2010 - 2018, encompassing 75000 fire brigade interventions on the area of Poznań and the Poznań county, constituted the source of the information.

The aim of the research was to create the set of cartographic visualizations demonstrating the rescuing and firefighting actions of the fire brigade in the form of various mapping methods, diagrams and other types of graphic presentation that allow one to observe links, relations and tendencies with regard to the occurrence of events that pose a threat to life, health or property.

The methods of multicriterial classification of qualitative and quantitative data, as well as gradual transformations of mapping techniques of data presentation, were employed in the research, resulting in $2 \mathrm{D}$ and $3 \mathrm{D}$ visualizations oriented toward the opportunity to analyze data from the intervention base in terms of the event type, location and time of occurrence. Apart from traditional mapping techniques, more complex and new graphic solutions, such as the rose graph, tessellation grid map, time-event prism map or indexed zonal statistic map, were utilized. Such combination is supposed to facilitate the analysis and interpretation of the data collected in the intervention base.

The evaluation of those visualization methods in terms of their effectiveness and usefulness, carried out with the help of experts, i.e. fire brigade members, brought about some interesting conclusions. The set of graphic solutions targeted at conducting analyses on operational data concerning the actions of the fire brigade, as well as the knowledge about the effectiveness of the visualizations worked out in the demonstration of spatial and temporal principles of the occurrence of events that required the fire brigade intervention on the examined area, constitute the results of the research. 